\title{
Chemotherapy against human African trypanosomiasis: Is there a road to success?
}

\author{
CHRISTIAN BURRI* \\ Swiss Tropical and Public Health Institute, Department of Medicines Research, Basel, Switzerland; University of Basel, \\ Basel, Switzerland
}

(Received 31 May 2010; revised 1 fuly 2010; accepted 2 fuly 2010; first published online 20 October 2010)

SUMMARY

For over fifty years, human African trypanosomiasis (HAT, sleeping sickness) has been treated with suramin, pentamidine and the very toxic organo-arsenical melarsoprol that was the only drug available for effective treatment of the second stage of the disease. Recently there have been significant efforts using molecular and biochemical approaches to drug design, including high-throughput screening, but the number of lead compounds with promising activity against $T$. brucei $\mathrm{spp}$. and an acceptable toxicity index has remained astonishingly small. Clinical research continues to be difficult due to the economic constraints and the complexity of trials on a low prevalence disease in remote and impoverished African regions. Despite those limitations the situation for the patients is improving thanks to the combination of a number of critical factors. By the late 1990s the disease had reached epidemic levels that triggered political support. WHO would sign a donation agreement with the manufacturers for all drugs to treat HAT. A result of this agreement was that eflornithine which is much safer than melarsoprol became available and widely used by non-governmental organizations. The IMPAMEL I and II programmes demonstrated that against all odds the conduct of clinical trials on HAT was feasible. This allowed the initiation of trials on combination therapies which eventually resulted in the nifurtimox-eflornithine combination treatment (NECT). This combination is currently being introduced as first line treatment, and there is even the prospect of having a new compound, fexinidazole, in the development pipeline. This review summarizes the key information about the existing drugs and gives a comprehensive summary about the recent and currently ongoing efforts towards new drugs.

Key words: Human African trypanosomiasis, sleeping sickness, treatment, drugs, pentamidine, suramin, melarsoprol, eflornithine, NECT, pafuramidine maleate, DB289, fexinidazole.

\section{INTRODUCTION}

The options for treatment of sleeping sickness are very limited and the selection of the appropriate drug for treatment is determined primarily by the disease stage and the causative pathogen, but in developing countries is also dependent on the logistics and technical level of the treatment facility. Presently, all drugs for treatment of human African trypanosomiasis (HAT) are donated to the WHO by the manufacturers sanofi-aventis and Bayer in the framework of a three-point agreement of adequate medicine supplies, disease surveillance and management, plus research and development for new treatments (Bayer, 2002; IFPMA and International Federation of Pharmaceutical Manufacturers and Associations, 2008). The drugs can be ordered by authorized national programmes or NGOs at the cost of shipment. An encouraging result of the agreement is that eflornithine, which is much safer than melarsoprol, is again available and has become widely used by

* Corresponding author: Christian Burri, MPharm. Ph.D., Head, Department of Medicines Research, Swiss Tropical \& Public Health Institute, Socinstrasse 57, P.O. Box, CH-4002 Basel, Switzerland. Tel: +4161225 26 61. Fax: +41612252678. E-mail: Christian.Burri@unibas.ch non-governmental organizations (Chappuis, 2007) and increasingly also by national control programmes. Nevertheless, well tolerated and simple to use medicines to treat the disease are needed. The way towards this goal has been a narrow and bumpy track in the past, but there are clear signs for a significant improvement. This review summarizes the key facts about the existing drugs and gives a comprehensive summary of the recent and currently ongoing efforts towards new drugs.

\section{PENTAMIDINE}

Pentamidine, currently available as pentamidine isethionate, Pentacarinat ${ }^{\circledR}$ (sanofi-aventis) in $200 \mathrm{mg}$ vials for intramuscular injection, is the drug of choice for treatment of first stage HAT caused by $T$. b. gambiense. The standard dosage regimen is $4 \mathrm{mg} \mathrm{kg}{ }^{-1}$ body weight per day for seven days. Because of the risk of hypotension after intravenous (i.v.) application the drug is usually given as deep intramuscular (i.m.) injection. In settings where good nursing care and monitoring are available, an intravenous infusion in saline over two hours may be used as an alternative. The recommended dose calculation for pentamidine has shifted over time from the base 
to the salt moiety resulting in a significant reduction of the active molecule injected when Pentacarinat ${ }^{\circledR}$ is given compared to the formerly used Lomidine ${ }^{\circledR}$ (Dorlo and Kager, 2008). Whereas this reduction seems to have an effect on the treatment efficacy of cutaneous leishmaniasis, the efficacy of pentamidine in HAT continues to be excellent and no change of the current practice, but rather a formal adaptation of the recommendation seems appropriate. The stable very low relapse rate, despite the extensive former use in prophylaxis programmes (Waddy, 1970), may be due to the uptake of the molecule by three different transporters (Barrett et al. 2007). Pharmacokinetic evidence indicates that three injections may be equally effective (Bronner et al. 1991; Bronner, 1994) and a respective comparative clinical trial is currently ongoing to evaluate this (ISRCTN55042030). Due to the high potency of pentamidine and the low drug levels detected in the CSF (Bronner et al. 1991) the use to treat 'intermediate stage' patients (up to 10 or 20 white blood cells (WBC) $\mathrm{mm}^{-3}$ in CSF) was suggested (Doua et al. 1996). The resulting efficacy in respective studies is equivocal (Ruiz et al. 2002; Lejon et al. 2003; Balasegaram et al. 2006b). Hence the use of pentamidine for patients with 5-20 cells $\mathrm{mm}^{-3}$ should not be generally recommended, but should be restricted to areas where melarsoprol is still in use, and where a very good adherence to follow up and rapid access to rescue treatment is guaranteed.

Compared to the drugs used for treatment of second stage HAT, pentamidine is well tolerated and most reactions are reversible. In the treatment of HAT by i.m. injection, site pain and transient swelling, abdominal pain, diarrhoea, nausea, vomiting and hypoglycaemia $(5-40 \%)$ are the most frequently reported adverse events (Médecins Sans Frontières, 2007). Other important adverse drug reactions (ADRs) like leucopenia, thrombocytopenia, hyperpotassaemia and QT-prolongation which are seen in treatment of other diseases e.g. Pneumocystis carinii (Anonymous, 2008), are only rarely reported in HAT probably due to the lack of adequate tools for patient monitoring. In addition, hyperglycaemia was reported $(5 \%)$, very rarely with persistent manifestation of diabetes (Doua and Yapo, 1993).

SURAMIN

Suramin, introduced in 1920, is the oldest trypanocidal medicine and one of the oldest drugs still in use along with quinine and aspirin, for instance. It is marketed as Germanin ${ }^{\circledR}$ (Bayer) in ampoules of $1 \mathrm{~g}$ and is effective against the first stage of both forms of the disease. For treatment of $T$. b. gambiense pentamidine is generally preferred today because of a claimed higher efficacy and because suramin is also highly active against Onchocerca spp. with the risk of strong allergic reactions in areas where this parasite is present.
The most commonly used dosage regimen consists of a test dose of $4-5 \mathrm{mg} \mathrm{kg}^{-1}$ body weight at day 1 , followed by five injections of $20 \mathrm{mg} \mathrm{kg}^{-1}$ body weight i.v. every 3-7 days (e.g. days 3, 5, 12, 19, 26) of suramin (WHO, 1986) with a maximum dose per injection of $1 \mathrm{~g}$. The compound deteriorates rapidly in air and should be injected immediately after dilution in distilled water (Gustafsson et al. 1987). No new research on a more concise treatment schedule has been initiated despite a recommendation by the WHO TDR Scientific Working Group on African Trypanosomiasis of 2001.

Some degree of kidney damage is common, but nephrotoxicity is usually mild and reversible. The first symptoms of renal impairment are albuminuria, later cylinduria and haematuria. Other adverse drug reactions reported are early hypersensitivity reactions occurring in $0 \cdot 1-0 \cdot 3 \%$ of cases causing nausea, circulatory collapse and urticaria, and rare late hypersensitivity reactions such as exfoliative dermatitis and haemolytic anaemia, peripheral neuropathy, and bone marrow toxicity with agranulocytosis, thrombocytopenia (Burri and Brun, 2008).

Suramin has one of the longest half-lives of all drugs applied to humans; depending on the dosage schedule half-lives of 44-92 days were reported. $99.7 \%$ of the drug is bound to plasma proteins, which places suramin in the class of the most extensively bound drugs (Burri et al. 2004). Several different proteins have been reported to be involved (e.g. albumin, globulins, fibrinogen). Such an extensive binding intrinsically bears the risk of adverse reactions through displacement from its binding by interacting drugs.

It is not known if suramin resistant $T . b$. gambiense or $T$. $b$. rhodesiense strains exist today. Observed relapses could be attributed to incorrectly diagnosed $2^{\text {nd }}$ stage infections.

\section{MELARSOPROL}

The organo-arsenic compound melarsoprol, Arsobal $^{\circledR}$ (sanofi-aventis) has until very recently been the most widely used drug for treatment of second stage HAT caused by $T$. b. gambiense in resourcelimited countries. For this form of the disease, melarsoprol will become progressively replaced by the Nifurtimox-Eflornithine Combination therapy (NECT) treatment (see below), but it is still the only choice for treatment of T. b. rhodesiense HAT. For $T$. b. gambiense an abridged treatment schedule of 10 injections $\left(2 \cdot 2 \mathrm{mg} \mathrm{kg}^{-1}\right.$ bodyweight per day) on consecutive days was recommended by the International Scientific Council for Trypanosomiasis Research and Control (ISCTRC) in 2004 (Schmid et al.2005). For treatment of T. b. rhodesiense various lengthy and complicated treatment schedules involving three series of three injections on consecutive days, with intervening rest periods of seven days, 
sometimes preceded by single injections of suramin, are still used (Burri and Brun, 2008). Research to assess the 10-day schedule in $T$. b. rhodesiense was very recently completed (see clinical research and outlook section below) and the same abridged treatment schedule of 10 injections $\left(2 \cdot 2 \mathrm{mg} \mathrm{kg}^{-1}\right.$ bodyweight per day) on consecutive days was recommended by the $30^{\text {th }}$ ISCTRC 2009 in Kampala (official publication pending).

Hospitalization during treatment with melarsoprol is mandatory because of the high rate of adverse drug reactions which may be severe or life threatening. The most important one is an encephalopathic syndrome (ES) which occurs very variably in an average of $4.7 \% \quad$ T. $\quad$ b. gambiense and $8.0 \%$ $T$. $b$. rhodesiense patients, with a case fatality rate of $44 \%$ and $57 \%$ respectively (Seixas, 2004). An immunological basis for ES has long been suspected and recent investigations indicating that a small number of alleles of the human leukocyte antigen (HLA) were associated with a significantly increased risk for ES have corroborated this hypothesis (Seixas, personal communication). The management of ES remains very difficult and there is no good scientific evidence for the best approach. Currently the use of dexamethasone, diazepam and where possible the maintenance of the physiological balance are recommended (Médecins Sans Frontières, 2007). The use of mannitol and adrenalin, which was habitual for a long time, was shown to be associated with a high mortality in a recently conducted study (Seixas, unpublished data). Recently, the successful experimental use of a substance $\mathrm{P}$ receptor antagonist, aprepitant $\left(\mathrm{Emend}^{\circledR}, \mathrm{MSD}\right)$ in rats to reduce the CNS neuroinflammatory reaction, was reported (Rodgers et al. 2007). Other reported severe adverse reactions are peripheral motoric (palsy) or sensorial (paresthesia) neuropathies (Van Nieuwenhove, 1999). Recent investigations showed that treatmentinduced ECG alterations were frequent, but clinically irrelevant (Blum et al. 2007). Skin reactions like pruritus and maculopapular eruptions are fairly common, but severe complications like bullous eruptions only occur in less than 1\% (WHO, 1998; Schmid et al. 2005). The irritating and painful effects of the injections, often leading to thrombophlebitis can be mitigated by a good injection technique and the use of new or sharp injection needles.

In several foci of Angola, the Democratic Republic of the Congo (DRC), Southern Sudan and Uganda, treatment failures have reached levels of $30 \%$ of those treated. Several parasites isolated from relapse cases in the field lack the P2 transporter which is necessary to import melarsoprol into the parasite (summarized in Barrett et al. 2007). However, so far the demonstration of parasite resistance in the field has been hampered by difficulties in retrieving T. b. gambiense isolates from patients for investigation.

\section{EF LORNITHINE}

Eflornithine, Ornidyl ${ }^{\circledR}$ (sanofi-aventis), is the only molecule which has been registered against HAT in the past 50 years. Several studies comparing melarsoprol and eflornithine showed a clearly reduced mortality and cumulative incidence of relapses under eflornithine and the drug is therefore recommended as first line treatment for second stage $T . b$.gambiense HAT (Chappuis et al. 2005; Balasegaram et al. 2006a; Checchi et al. 2007), but the use of eflornithine against $T . b$. rhodesiense is not advised because of the innately reduced susceptibility of this parasite due to a higher ornithine decarboxylase turnover (Iten et al. 1997). The most commonly used dosage regimen for the treatment of $T$. b. gambiense HAT consists of $100 \mathrm{mg} \mathrm{kg}^{-1}$ body weight at intervals of $6 \mathrm{~h}$ for 14 days of eflornithine given as short infusions (Burri and Brun, 2008). An abridged 7-day treatment regimen can not be generally recommended due to the significantly lower efficacy (Pepin et al. 2000).

Adverse drug reactions during eflornithine therapy are frequent and the characteristics are similar to other cytotoxic drugs for the treatment of cancer. Their occurrence and intensity increase with the duration of treatment and the severity of the general condition of the patient. Generally, adverse drug reactions to eflornithine are reversible after the end of treatment. They include convulsions (7\%), gastrointestinal symptoms like nausea, vomiting and diarrhoea $(10 \%-39 \%)$, and bone marrow toxicity leading to anaemia, leucopenia and thrombocytopenia (25-50\%). In experimental cancer treatment with considerably longer therapeutic schedules hearing impairment (5\%) and alopecia (5-10\%) were also reported (Burri and Brun, 2003).

Eflornithine is very expensive and only the donation programme has allowed its increasing use in endemic countries. The introduction of eflornithine as first line treatment replacing melarsoprol in treatment facilities operated by national control programmes is only progressing slowly. This is mainly due to the complexity of eflornithine administration, requiring 56 short infusions over two weeks and the associated substantial logistic implications and indirect costs (e.g. acquisition and transport of infusion materials from the control programme base to the field), and the need for sufficient and trained human resources to maintain adequate round-the-clock nursing care. Since 2007 the WHO, with funding from the sanofi-aventis programme, has provided eflornithine in kits including all necessary ancillary materials (Priotto et al. 2008), but considerable logistic constraints remain as the kit for two patients has a volume of about $1 \mathrm{~m}^{3}$.

An oral formulation was thought to solve many of the treatment related difficulties, but was abandoned after discouraging results of a pharmacokinetic trial (Na-Bangchang et al. 2004; Jansson et al. 2008). 
Another serious concern with eflornithine monotherapy is the probability of the development of resistance, which would have catastrophic consequences. The first reports of a decreasing efficacy of eflornithine in certain centres in Southern Sudan seem to have already emerged (Dr. P. Simarro, WHO, personal communication). It is thus vital to protect the efficacy of this drug by using it in combination regimens as soon as possible.

\section{CLINICAL RESEARCH AND OUTLOOK}

Clinical research on new drugs for treating HAT continues to be limited for various reasons. Following economic logic, the interest of commercial corporations in the development of new drugs against this disease only affecting extremely poor populations is limited. Despite largely increased molecular, biochemical and screening efforts, the number of lead compounds with promising activity against $T$. bruce $i$ spp. and an acceptable toxicity index has remained astonishingly small. Last but not least, clinical trials to assess the safety and the efficacy of new drugs are extremely difficult to conduct due to the low prevalence, the remoteness of the patients and the very long follow up time. Hence, the currently available options for the treatment are suboptimal for lack of efficacy and toxicity, and resistance is a latent threat due to the exclusive use of monotherapies. For those reasons, recent efforts have focused on optimizing the therapeutic regimens and on developing combination therapy using the registered drugs or those used to treat related diseases.

\section{IMPAMEL III (Improved Application of Melarsoprol)}

The abridged treatment schedule of 10 injections $\left(2 \cdot 2 \mathrm{mg} \mathrm{kg}^{-1}\right.$ bodyweight per day) of melarsoprol had been recommended for treatment of second stage T. b. gambiense HAT by the ISCTRC in 2004 after the completion of the IMPAMEL I and II programmes. The evaluation of the schedule against $T$. $b$. rhodesiense of the schedule was very recently completed (IMPAMEL III trials). Due to the very small total patient number and their remote location, no pivotal trials are feasible. Therefore, a proofof-concept trial $(\mathrm{n}=60)$ and a utilization study $(n=78)$ using historic controls as comparator were sequentially carried out. The incidence of encephalopathic syndromes in the trial population was $11 \cdot 2 \%$ (CI 5-17\%) versus 13\% (CI 9-17\%) in the historic data. The respective case fatality rates were $8.4 \%$ (CI 3-13.8\%) and 9.3\% (CI 6-12.6\%). All patients discharged alive were free of parasites at the end of treatment. Six months after discharge $99 \%$ of patients were considered clinically cured. The total hospitalization time was reduced from 29 to 13 days (Kuepfer, 2009). This first improvement of the treatment after 60 years bears considerable socioeconomic advantages, but this cannot hide the fact that new and adequate drugs are urgently needed to treat this form of the disease.

\section{$N E C T$}

An alternative compound which was considered in the context of reassessment of existing drugs is nifurtimox (Lampit ${ }^{\circledR}$, Bayer), which was introduced for treatment of Chagas disease (T. cruzi) in the late 1960 s. It is not registered to treat African trypanosomiasis but had been experimentally used with equivocal outcomes and became recently available for compassionate treatment in combination with other trypanocidal drugs of patients not responding to melarsoprol (Priotto et al. 2006). Different empirically derived treatment schedules were used; for combination with other trypanocidal drugs usually $15 \mathrm{mg} \mathrm{kg}^{-1}$ per day for 10 days by oral route was selected. Nifurtimox is generally not well tolerated, and only about one-third of the patients remain free from adverse drug reactions but generally adverse effects are not severe, very rarely fatal, and are dose related. Gastrointestinal disturbances with nausea, abdominal pains and vomiting are very frequent, and neurological adverse reactions with general convulsions, tremor or agitation may occur. The development of peripheral polyneuropathy and generalized skin reactions were seen as occasional events (Burri and Brun, 2008). The frequency of adverse reactions increases with therapy duration and all were rapidly reversible after discontinuation of the drug (Pepin et al. 1992).

Several trials assessing combinations of eflornithine, melarsoprol and nifurtimox have been conducted. In all trials, the efficacy was better in the combination arms compared to the monotherapies. However, combinations containing melarsoprol resulted in very high frequencies of severe adverse drug reactions (Priotto et al. 2006; Bisser et al. 2007). Eventually, a multi-centre trial, NECT - nifurtimoxeflornithine combination therapy, was initiated in the Republic of Congo and the DRC comparing NECT with the standard eflornithine therapy (Chappuis, 2007). The treatment consisted of eflornithine $200 \mathrm{mg} \mathrm{kg}^{-1}$ i.v. short infusion every $12 \mathrm{~h}$ for 7 days plus nifurtimox $15 \mathrm{mg} \mathrm{kg}^{-1}$ orally per day $\left(5 \mathrm{mg} \mathrm{kg}^{-1}\right.$ every $8 \mathrm{~h}$ ) for 10 days. This represents a considerable simplification compared to the standard eflornithine schedule: The number of infusions can be reduced from 56 to 14 , it shortens hospitalization time by one third and reduces the total amount of eflornithine by half (Priotto et al. 2007). The inventors took a substantial risk in the dose determination, because eflornithine has a very short half-life requiring four daily drug applications and because a seven days short-course eflornithine 
treatment previously led to significantly lower efficacy (Pepin et al. 2000). It was argued that the short half-life of eflornithine might be balanced by the long-lasting pharmacodynamic effect on trypanosomes, explained by the long time (18-19 h) needed by $T$. b. gambiense to replenish their ornithine decarboxylase after inhibition by eflornithine (Priotto et al. 2007) and that a previous combination trial using standard dose eflornithine with nifurtimox had resulted in very low relapse rate (Priotto et al. 2006). A total of 286 patients were enrolled, the relapse rate after 18 months of follow up was $5 \cdot 7 \%$ under eflornithine and $1.4 \%$ under NECT treatment (intention to treat population, i.e. all randomised patients, apart from one who absconded on day 1). Adverse events were frequent in both groups; $41(28.7 \%)$ patients in the eflornithine group and $20(14.0 \%)$ in the NECT group had major (grade 3 or 4) reactions (Priotto et al. 2009). A smaller scale trial with a similar design was conducted in two treatment centres in Uganda (ISRCTN03148609). Only very generic data have been made available so far, but these support a positive evaluation of the NECT treatment (Kansiime et al. 2009). NECT has large advantages over the eflornithine monotherapy as it is easier to administer, reduces the necessary drug, staff and logistic resources, requires a significantly shorter hospital stay and it may be predicted to have a positive effect against development of drug resistance. Those features are of particular importance considering the rural character of the treatment centres. Based on the favourable results of the trials conducted so far, an application for the inclusion of nifurtimox, to be used in combination with eflornithine, for treatment of second stage T. b. gambiense HAT into WHO's Essential Medicines List (EML) was submitted by the Drugs for Neglected Diseases Initiative (DNDi) and was granted in May 2009 (DNDi, 2009). A study evaluating the tolerability, feasibility, and effectiveness of NECT in 'real-life' conditions was initiated simultaneously (NECT-FIELD) (DNDi, 2008a) and within one year the planned 620 patients had been enrolled in the DRC (C. Schmid, personal communication). By March 2010, NECT had been introduced in Central African Republic, Chad, DRC, Equatorial Guinea, Sudan and Uganda (DNDi, 2010).

This momentum should now be used to submit the NECT schedule to studies in the $T$. $b$. rhodesiense vervet monkey model. Using a futility approach, a decision whether in-depth studies in human are justified or not could be relatively rapidly made. Based on the pharmacology of the drugs (see above) the likelihood for success with $T$. b. rhodesiense is limited, but as this will be the only potential new approach to treat this form of the diseases for a long time, the effort would be justified.

\section{DB289}

The development of new medicines against HAT has recently experienced a serious setback, when the only new drug in clinical assessment failed at the very end of the development programme. The international Consortium for Parasitic Drug Development (CPDD) led by the University of North Carolina, Chapel Hill, USA, received funding by the Bill and Melinda Gates Foundation (BMGF) to discover and bring selected molecules to registration. DB289 (pafuramidine maleate), an orally active prodrug had proven considerable trypanocidal activity and low toxicity in pre-clinical testing and was chosen for further development in the year 2000. The compound underwent extensive Phase I (healthy volunteers) and Phase II testing (proof of concept in patients). A pivotal Phase III trial was initiated in August 2005 and its follow-up ended in mid-2009. 273 patients were enrolled in four centres in the DRC and in one centre each in Angola and South Sudan. All patients completed the assigned study drug regimen of pafuramidine $100 \mathrm{mg}$ twice a day oral for 10 days or pentamidine $4 \mathrm{mg} \mathrm{kg}^{-1} \mathrm{i} . \mathrm{m}$. for 7 days. After unblinding, the safety profile of pafuramidine appeared to be inconspicuous; ALT and AST elevations were frequently recorded but only reached Grades 2 and 3 elevation under pentamidine therapy. The per protocol efficacy at the 12-months follow up was $89 \%$ for pafuramidine and $96 \%$ for pentamidine, respectively (Pohlig et al. 2008).

An additional Phase I study in which healthy volunteers received pafuramidine $100 \mathrm{mg}$ BID for 14 days was conducted in the last quarter of 2007. The study was designed to provide supportive safety data for the registration of pafuramidine for sleeping sickness and pneumocystis pneumonia. The pafuramidine development programme was placed on clinical hold by the US FDA to allow the investigation of unexpected liver toxicity observed post-treatment to this trial. Subsequently, 5 subjects of the same healthy volunteer study developed renal insufficiency approximately 8 weeks post- treatment that required medical intervention. Re-examination of the Phase III sleeping sickness data identified 3 subjects who had developed glomerulonephritis/nephropathy post-pafuramidine treatment; 2 of these events may retrospectively be considered possibly related to pafuramidine. No patient in the pentamidine group was reported to have renal disease. The clinical development programme for pafuramidine was discontinued at this time (Pohlig et al. 2008).

\section{New Drugs}

Efforts to identify drug targets, and in high throughput as well as live cell screening have been substantially increased and funded since the turn of the century. A number of new drug targets have been 
identified and numerous compounds with promising activity in preliminary tests identified. So far, drug target validation and lead identification and optimization have proved to be difficult, and consequently there are currently only a very few molecules in preclinical and only one in clinical development for treatment of HAT. The most advanced new drug in the pipeline is fexinidazole which belongs to the nitroimidazole class of drugs which has previously shown activity against trypanosomes. The molecule was re-discovered during an extensive compound mining effort undertaken by the DND $i$ since 2005 to explore new and old nitroimidazoles as drug leads against human African trypanosomiasis (HAT). The substance proved to be orally active against $T . b$. gambiense and T. $b$. rhodesiense in animal studies and had an excellent safety profile. Since it penetrates the BBB it could be effective in both stages of sleeping sickness. In September 2009 the firstin-human Phase I study was initiated (DNDi, 2008b; Anonymous, 2009).

Whatever the number and quality of compounds emerging from the discovery process, their clinical evaluation will remain critically difficult. The case of pafuramidine maleate has demonstrated that new HAT drugs can be developed according to international guidances and under the scrutiny of a first tier regulatory agency (i.e. FDA). However, to conduct the necessary trials in rural, extremely resourcelimited and often insecure areas require substantial effort. Should the positive downwards trend in the patient number (WHO, 2006) continue, the conduct of Phase II and III trials will even become a more difficult and complex multi-site and multi-country task. This will require substantial and continuous funding, and it will be essential that where more than one lead molecule is identified research is not progressed in a competitive manner. Instead, cooperation and state-of-the-art trial design would be demanded.

One of the major weaknesses of the clinical trials conducted on HAT so far is the variety of definitions for relapses, treatment failures and cure rates used. This makes it virtually impossible to compare the efficacy results between trials and to perform metaanalysis. The WHO recently issued a reference document for the conduct of clinical trials in HAT (WHO, 2007). It is strongly recommended that future clinical trials on HAT should follow the definitions of this guideline to facilitate collaboration in the evaluation of new treatments and/or the comparison of data obtained by different groups. Standardization might become particularly important in the light of the previously mentioned further restrictions of HAT drug trials due to decreasing patient numbers.

The first large-scale clinical trial on HAT drugs, the IMPAMEL I trial comparing one of the lengthy standard schedules for treatment of second stage disease with melarsoprol with an abridged 10-day course, was initiated thirteen years ago (Burri et al. 2000). At this time there were virtually no activities in the field of HAT drug development, and the interest of the national sleeping sickness programmes in new treatments was very limited due to the very long tradition of complicated and toxic treatments and the perceived lack of any prospect for better drugs in the near future. The results of the IMPAMEL programme were somewhat frustrating as the encephalopathic syndromes could not been overcome, but it has pointed the direction in the conduct of HAT trials - and in the meantime we have travelled a long way. Right now, we seem to be about leaving the dirt track and a better engine to accelerate seems to have been fitted to our vehicle. After a difficult experience with eflornithine monotherapy, the nifurtimox-eflornithine combination treatment has a fair chance to supersede melarsoprol as first line treatment for second stage $T$. b. gambiense disease. Travelling on a decent road does not mean detours can be avoided as the example of DB289 has shown. At this point the perseverance from all those involved and continuous funding will be required as we still will have to deal with a long and winding road. Travelling ahead at the current pace should eventually lead us to the promised destination, e.g. to modern, easier to administer drugs, or even to drugs which may be simultaneously used against first and second stage disease and thus eliminate the trouble of lumbar punctures.

\section{REFERENCES}

Anonymous (2008). Pentacarinat ${ }^{\circledR}$. In ArzneimittelKompendium der Schweiz (ed. Dokumed A. G., Basel., Switzerland).

Anonymous (2009). Human African Trypanosomiasis: First in Man Clinical Trial of a New Medicinal Product, the Fexinidazole. ClinicalTrials.gov (Clinical Trials Registry) http://www.clinicaltrials.gov/ct2/show/ NCT00982904?term $=$ NCT00982904\&rank=1 (Accessed 24 June 2010).

Balasegaram, M., Harris, S., Checchi, F., Ghorashian, S., Hamel, C. and Karunakara, U. (2006a). Melarsoprol versus eflornithine for treating late-stage Gambian trypanosomiasis in the Republic of the Congo. Bulletin of the World Health Organization 84, 783-791.

Balasegaram, M., Harris, S., Checchi, F., Hamel, C. and Karunakara, U. (2006b). Treatment outcomes and risk factors for relapse in patients with early-stage human African trypanosomiasis (HAT) in the Republic of the Congo. Bulletin of the World Health Organization 84, 777-782.

Barrett, M. P., Boykin, D. W., Brun, R. and

Tidwell, R. R. (2007). Human African trypanosomiasis: pharmacological re-engagement with a neglected disease. British Yournal of Pharmacology 152, 1155-1171.

Bayer (2002). Bayer sustainable development report 2004. Vol. 2008. http://www.bayer.com/en/sustainable_ development_report_2004.pdfx (Accessed 29 July 2010). 
Bisser, S., N'siesi, F. X., Lejon, V., Preux, P. M., Van Nieuwenhove, S., Miaka Mia Bilenge, C. and Buscher, P. (2007). Equivalence trial of melarsoprol and nifurtimox monotherapy and combination therapy for the treatment of second-stage Trypanosoma brucei gambiense sleeping sickness. Fournal of Infectious Diseases 195, 322-329.

Blum, J. A., Burri, C., Hatz, C., Kazumba, L., Mangoni, P. and Zellweger, M. J. (2007). Sleeping hearts: the role of the heart in sleeping sickness (human African trypanosomiasis). Tropical Medicine and International Health 12, 1422-1432.

Bronner, U. (1994). Pharmacokinetics of pentamidine. Focus on treatment of Trypanosoma gambiense sleeping sickness. PhD Thesis, Karolinska Institute, Stockholm.

Bronner, U., Doua, F., Ericsson, O., Gustafsson, L., Miezan, T., Rais, M. and Rombo, L. (1991). Pentamidine concentrations in plasma, whole blood and cerebrospinal fluid during treatment of Trypanosoma gambiense infection in Côte d'Ivoire. Transactions of the Royal Society of Tropical Medicine and Hygiene 85, 608-611.

Burri, C. and Brun, R. (2003). Eflornithine for treatment of human African trypanosomiasis. Parasitology Research 90 (Suppl), S49-S52.

Burri, C. and Brun, R. (2008). Chapter 76: Human African trypanosomiasis. In Manson's Tropical Diseases (eds. Cook, G., and Zumla, A.), pp. 1307-1325. W.B. Saunders, London.

Burri, C., Nkunku, S., Merolle, A., Smith, T., Blum, J. and Brun, R. (2000). Efficacy of new, concise schedule for melarsoprol in treatment of sleeping sickness caused by Trypanosoma brucei gambiense: a randomised trial. Lancet 355, 1419-1425.

Burri, C., Stich, A. and Brun, R. (2004). Current chemotherapy of Human African sleeping sickness. In The Trypanosomiases (eds. Maudlin, I., Holmes, P., and Miles, M. A.), pp. 403-419. CABI, Wallingford.

Chappuis, F. (2007). Melarsoprol-free drug combinations for second-stage Gambian sleeping sickness: the way to go. Clinical Infectious Diseases 45, 1443-1445.

Chappuis, F., Udayraj, N., Stietenroth, K., Meussen, A. and Bovier, P. A. (2005). Eflornithine is safer than melarsoprol for the treatment of second-stage Trypanosoma brucei gambiense human African trypanosomiasis. Clinical Infectious Diseases 41, 748-751.

Checchi, F., Piola, P., Ayikoru, H., Thomas, F., Legros, D. and Priotto, G. (2007). Nifurtimox plus Eflornithine for late-Stage sleeping sickness in Uganda: A case series. PLoS Neglected Tropical Diseases 1, e64.

DNDi (2008a). NECT: Next steps. In DNDi Newsletter, 17.

DNDi (2008b). Fexinidazole progresses into clinical development. DNDi Newsletter, 17.

DNDi (2009). NECT added to WHO essential medicines list as combination treatment against sleeping sickness. DNDi Press Release. http://www.dndi.org/pressreleases/456-nect-added-to-who-essential-medicineslist-as-combination-treatment-against-sleepingsickness.html (Accessed 24 June 2010)

DNDi (2010). NECT - Nifurtimox-Eflornithine: Co-Administration (HAT). http://www.dndi.org/ portfolio/nect.html (Accessed 24 June 2010)
Dorlo, T. P. and Kager, P. A. (2008). Pentamidine dosage: a base/salt confusion. PLoS Neglected Tropical Diseases 2, e225.

Doua, F., Miezan, T. W., Sanon Singaro, J. R., Boa Yapo, F. and Baltz, T. (1996). The efficacy of pentamidine in the treatment of early-late stage Trypanosoma brucei gambiense trypanosomiasis. American Fournal of Tropical Medicine and Hygiene 55, 586-588.

Doua, F. and Yapo, F. B. (1993). Human trypanosomiasis in the Ivory Coast - therapy and problems. Acta Tropica 54, 163-168.

Gustafsson, L. L., Beerman, B. and Aden Abdi, Y. (1987). Suramin. In Handbook of Drugs for Tropical Parasitic infections (eds. Gustafsson, L. L., Beerman, B., and Aden Abdi, Y.), pp. 160-163. Taylor \& Francis, Basingstoke.

IFPMA and International Federation of Pharmaceutical Manufacturers \& Associations (2008). Health Partnerships for the Developing World -Sanofi-Aventis Sleeping Sickness Program. Vol. 2008.

Iten, M., Mett, H., Evans, A., Enyaru, J. C., Brun, R. and Kaminsky, R. (1997). Alterations in ornithine decarboxylase characteristics account for tolerance of Trypanosoma brucei rhodesiense to D,L-alphadifluoromethylornithine. Antimicrobial Agents and Chemotherapy 41, 1922-1925.

Jansson, R., Malm, M., Roth, C. and Ashton, M. (2008). Enantioselective and nonlinear intestinal absorption of eflornithine in the rat. Antimicrobial Agents and Chemotherapy 52, 2842-2848.

Kansiime, F., Adibaku, S., Wanboga, C., Iddi, F., Matovu, E., Yamuah, L. and Kioy, D. (2009). Clinical study of nifurtimox-eflornithine combination treatment (NECT) of late stage Trypanosoma brucei gambiense patients in NW Uganda: Implications for the National sleeping sickness control programme. In 30th Meeting of the International Scientific Council for Trypanosomiasis Research and Control (ISCTRC) (ed. OAU/STRC, Nairobi), p.69, Kampala, Uganda.

Kuepfer, I. (2009). Impamel III - Improved melarsoprol application for the treatment of second stage T. b. rhodesiense sleeping sickness. In 30th Meeting of the International Scientific Council for Trypanosomiasis Research and Control (ISCTRC) (ed. OAU/STRC, Nairobi), p. 70. Kampala, Uganda.

Lejon, V., Legros, D., Savignoni, A., Etchegorry, M. G., Mbulamberi, D. and Buscher, P. (2003). Neuro-inflammatory risk factors for treatment failure in "early second stage" sleeping sickness patients treated with Pentamidine. Fournal of Neuroimmunology 144, 132-138.

Médecins Sans Frontières (2007). Clinical Guidelines. http://www.refbooks.msf_docs/en/Clinical_Guide/ CG_en.pdf (Accessed 29 July 2010).

Na-Bangchang, K., Doua, F., Konsil, J., Hanpitakpong, W., Kamanikom, B. and Kuzoe, F. (2004). The pharmacokinetics of eflornithine (alphadifluoromethylornithine) in patients with late-stage T. b. gambiense sleeping sickness. European Fournal of Clinical Pharmacology 60, 269-278.

Pepin, J., Khonde, N., Maiso, F., Doua, F., Jaffar, S., Ngampo, S., Mpia, B., Mbulamberi, D. and 
Kuzoe, F. (2000). Short-course eflornithine in Gambian trypanosomiasis: a multicentre randomized controlled trial. Bulletin of the World Health Organization 78, 1284-1295.

Pepin, J., Milord, F., Meurice, F., Ethier, L., Loko, L. and Mpia, B. (1992). High-dose nifurtimox for arseno-resistant Trypanosoma brucei gambiense sleeping sickness: an open trial in central Zaire. Transactions of the Royal Society of Tropical Medicine and Hygiene 86, 254-256.

Pohlig, G., Bernhard, S., Blum, J., Burri, C., Mpanya Kabeya, A., Fina Lubaki, J.-P., Mpoo Mpoto, A., Fungula Munungu, B., Kambau Manesa Deo, G., Nsele Mutantu, P., Mbo Kuikumbi, F., Fukinsia Mintwo, A., Kayeye Munungi, A., Dala, A., Macharia, S., Miaka Mia Bilenge, C., Kande Betu Ku Mesu, V., Ramon Franco, J., Dieyi Dituvanga, N. and Olson, C. (2008). Phase 3 trial of pafuramidine maleate (DB289), a novel, oral drug, for treatment of first stage sleeping sickness: Safety and Efficacy. In 57th Meeting of the American Society of Tropical Medicine and Hygiene pp. Abstract No. 542. New Orleans.

Priotto, G., Fogg, C., Balasegaram, M., Erphas, O., Louga, A., Checchi, F., Ghabri, S. and Piola, P. (2006). Three drug combinations for late-stage Trypanosoma brucei gambiense sleeping sickness: A randomized clinical trial in Uganda. PLoS Clinical Trials 1, e39: 31-38.

Priotto, G., Kasparian, S., Mutombo, W., Ngouama, D., Ghorashian, S., Arnold, U., Ghabri, S., Baudin, E., Buard, V., KazadiKyanza, S., Ilunga, M., Mutangala, W., Pohlig, G., Schmid, C., Karunakara, U., Torreele, E. and Kande, V. (2009). Nifurtimox-eflornithine combination therapy for second-stage African Trypanosoma brucei gambiense trypanosomiasis: a multicentre, randomised, phase III, non-inferiority trial. Lancet 374, 56-64.

Priotto, G., Kasparian, S., Ngouama, D., Ghorashian, S., Arnold, U., Ghabri, S. and Karunakara, U. (2007). Nifurtimox-Eflornithine combination therapy for second-stage Trypanosoma brucei gambiense sleeping sickness: A randomized clinical trial in Congo. Clinical Infectious Diseases 45, 1435-1442.

Priotto, G., Pinoges, L., Fursa, I. B., Burke, B., Nicolay, N., Grillet, G., Hewison, C. and
Balasegaram, M. (2008). Safety and effectiveness of first line eflornithine for Trypanosoma brucei gambiense sleeping sickness in Sudan: cohort study. British Medical Fournal 336, 705-708.

Rodgers, J., Bradley, B. and Kennedy, P. G. (2007). Combination chemotherapy with a substance $\mathrm{P}$ receptor antagonist (aprepitant) and melarsoprol in a mouse model of human African trypanosomiasis. Parasitology International 56, 321-324.

Ruiz, J. A., Simarro, P. P. and Josenando, T. (2002). Control of human African trypanosomiasis in the Quicama focus, Angola. Bulletin of the World Health Organization 80, 738-745.

Schmid, C., Richer, M., Bilenge, C. M., Josenando, T., Chappuis, F., Manthelot, C. R., Nangouma, A., Doua, F., Asumu, P. N., Simarro, P. P. and Burri, C. (2005). Effectiveness of a 10-day melarsoprol schedule for the treatment of late-stage human African trypanosomiasis: Confirmation from a multinational study (Impamel II). Fournal of Infectious Diseases 191, 1922-1931.

Seixas, J. (2004). Investigations on the encephalopathic syndrome during melarsoprol treatment of human African trypanosomiasis. PhD Thesis, Instituto de Higiene e Medicina Tropical, Universidade Nova de Lisboa.

Van Nieuwenhove, S. (1999). Present strategies in the treatment of human African trypanosomiasis. In Progress in Human African trypanosomiasis, Sleeping Sickness (eds. Dumas, M., Bouteille, B., and Buguet, A.), pp. 253-280. Springer, Paris.

Waddy, B. B. (1970). Chemoprophylaxis of human trypanosomiasis. In The African Trypanosomiases (ed. Mulligan, H. W.), pp. 711. Allan \& Unwin, London.

WHO (1986). Epidemiology and control of African trypanosomiasis. WHO Technical Report Series, 739.

WHO (1998). Control and surveillance of African trypanosomiasis, WHO, Geneva.

WHO (2006). Human African trypanosomiasis (sleeping sickness): epidemiological update. Weekly Epidemiological Report, 8, 71-80.

WHO (2007). Recommendations of the informal consultation on issues for clinical product development for human African trypanosomiasis. WHO/CDS/NTD/IDM/ 2007.1. Geneva, Switzerland. 